\title{
Synthesis of New Thiazole, Bithiazolidinone and Pyrano[2,3-d]thiazole Derivatives as Potential Antimicrobial Agents
}

Mohamed A. Salem ${ }^{1,2}$

\footnotetext{
1 Department of Chemistry, Faculty of Science, Al-Azhar University, 11284 Nasr City, Cairo, Egypt

2 Department of Chemistry, Faculty of Science and Arts, King Khalid University, Mohail Assir, KSA

Author's e-mail address: m_eltayyeb@hotmail.com
}

RECEIVED: July 7, 2016 * REVISED: October 7, $2016 *$ ACCEPTED: October 7, 2016

Abstract: In an attempt to find a new class of antimicrobial agents, a series of thiazole derivatives containing furan moiety $3,7,8 a, b, 10 a-c$ and 12 were prepared via the reaction of 2-cyano- $N$-(furan-2-ylmethyl)acetamide (1) with phenyl isothiocyanate and $\alpha$-halocarbonyl compounds. Also, many bithiazolidinone and pyranothiazole derivatives were synthesized through interaction of thiazolidinone 7 with appropriate electrophilic reagents. These compounds were screened for their antibacterial and antifungal activity. Among the synthesized compounds, thiazole derivatives $23 \mathrm{a}$ and $23 \mathrm{~b}$ were equipotent to gentamycin against $K$. pneumonia (MIC $0.49 \mu \mathrm{g} / \mathrm{mL}$ ), and showed in vitro antifungal activity equipotent to amphotericin B versus A. fumigatus (MIC $0.98 \mu \mathrm{g} / \mathrm{mL}$ ), A. clavatus (MIC $0.98 \mu \mathrm{g} / \mathrm{mL}$ ) and G. candidium (MIC $0.49 \mu \mathrm{g} / \mathrm{mL}$ ). Also, bithiazolidinones 14 and 15 were equipotent to amphotericin B in inhibiting the growth of $A$. clavatus (MIC $0.98 \mu \mathrm{g} / \mathrm{mL}$ ) and G. candidium (MIC $0.49 \mu \mathrm{g} / \mathrm{mL}$ ). Structures of the newly synthesized compounds were established by elemental analysis and spectral data.

Keywords: thiazole, bithiazolidinone, pyranothiazole, antimicrobial activity.

\section{INTRODUCTION}

D ESPITE a large number of antibiotics and chemotherapeutics available for medical use, the emerging resistance to old and new antibiotics has created a substantial need for new classes of antimicrobial agents. A potential approach to overcome the problem of antibiotic resistance is to design innovative agents with different modes of action, so that no cross resistance with present drugs can occur. ${ }^{[1]}$

Thiazoles are a familiar group of heterocyclic compounds possessing a wide variety of biological activities, and their usefulness as medicines are well established. This later is also an integral part of all the available penicillins, which have revolutionized the therapy of bacterial diseases. ${ }^{[2]}$ Thiazoles have also attracted continuing interest because of their varied biological activities, ${ }^{[3]}$ which have found applications in the treatment of allergies, ${ }^{[4]}$ hypertension, ${ }^{[5]}$ inflammation, ${ }^{[6]}$ schizophre- nia, ${ }^{[7]}$ microbial infections, ${ }^{[8,9]}$ HIV infections, ${ }^{[10]}$ hypnotics ${ }^{[11]}$ and for the treatment of pain. ${ }^{[12]}$ They have been also used as fibrinogen receptor antagonists with antithrombotic activity ${ }^{[13]}$ and as new inhibitors of bacterial DNA gyrase B. ${ }^{[14]}$

Moreover, many biologically important derivatives of furan substituted at 2- and 5-positions are frequently observed in nature show broad-spectrum phytocidal, antibacterial and insecticidal activities ${ }^{[15,16]}$ and exhibit pharmacological properties which serves as antidepressant and anti-inflammatory agents. ${ }^{[17,18]}$

In view of the above-mentioned findings, and as a continuation of our effort to identify new candidates, ${ }^{[19-26]}$ which are crucial in designing new, potent, selective, and less toxic antimicrobial agents, its report herein the synthesis and antimicrobial evaluation of some novel structure hybrids incorporating both the thiazole ring system with furan moiety. This combination was suggested in an attempt to investigate the influence of such 


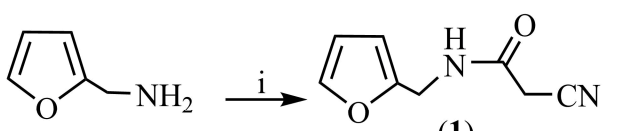

(1)

$$
\mathrm{i}=\mathrm{CNCH}_{2} \mathrm{COOEt}
$$

Scheme 1 . Synthesis of 2-cyano- $N$-(furan-2-ylmethyl)acetamide.

hybridization and structure variation on the anticipated biological activities, hoping to add some synergistic biological significance to the target molecules. The target compounds were rationalized so as to comprise some pharmacophores that are believed to be responsible for the biological activity of some relevant chemotherapeutic such as the carboxamido, amino and cyano groups functionalities. The substitution pattern of the thiazole ring was carefully selected in order to confer different electronic environment to the molecules.

\section{RESULTS AND DISCUSSION}

\section{Chemistry}

The starting compound 2-cyano- $\mathrm{N}$-(furan-2-ylmethyl)acetamide (1) was readily available from the solvent-free reaction of furan-2-ylmethanamine with an excess amount of ethyl cyanoacetate under fusion condition, [27] (Scheme 1).

The active methylene moiety of 2-cyano- $\mathrm{N}$-(furan-2ylmethyl)acetamide (1) was allowed to react with phenyl isothiocyanate in dimethylformamide in the presence of an equimolar amount of potassium hydroxide yielded the nonisolable intermediate potassium sulphide salt $\mathbf{2}$, which reacted in situ with chloroacetonitrile to afford the novel 4aminothiazole derivative 3 rather than thiophene structure 4. The reaction product $\mathbf{3}$ was confirmed on the basis of elemental analysis and spectral data. The IR spectrum of $\mathbf{3}$ showed absorption bands at 3423, 3308, 3110, 2184 and $1637 \mathrm{~cm}^{-1}$ assignable for $\mathrm{NH}_{2}, \mathrm{NH}, \mathrm{CN}$ and carboxamide groups, respectively. While its ${ }^{1} \mathrm{H}$ NMR spectrum showed doublet and singlet signals at $\delta 4.27,7.16 \mathrm{ppm}$ characteristic for $\mathrm{CH}_{2}$ and thiazole-H5 protons, doublet and triplet signals at $\delta 6.14,6.34 \mathrm{ppm}$ can be assigned to furan protons, in addition to singlet and triplet signals at $\delta 8.22$, $10.08 \mathrm{ppm}$ for $\mathrm{NH}_{2}$ and $\mathrm{NH}$ protons, respectively. Also, it has been found that, the in situ reaction of $\mathbf{2}$ with 2-chloro$N$-phenylacetamide afforded thiazolidin-4-one 7 rather than the expected thiophene 6 . The formation of thiazolidin-4-one 7 may be rationalized through the first $S$ alkylation to form non-isolable intermediate $\mathbf{5}$ followed by nucleophilic addition of $\mathrm{NH}$ group to the amidic carbonyl with elimination of aniline. ${ }^{1} \mathrm{H}$ NMR spectrum of compound

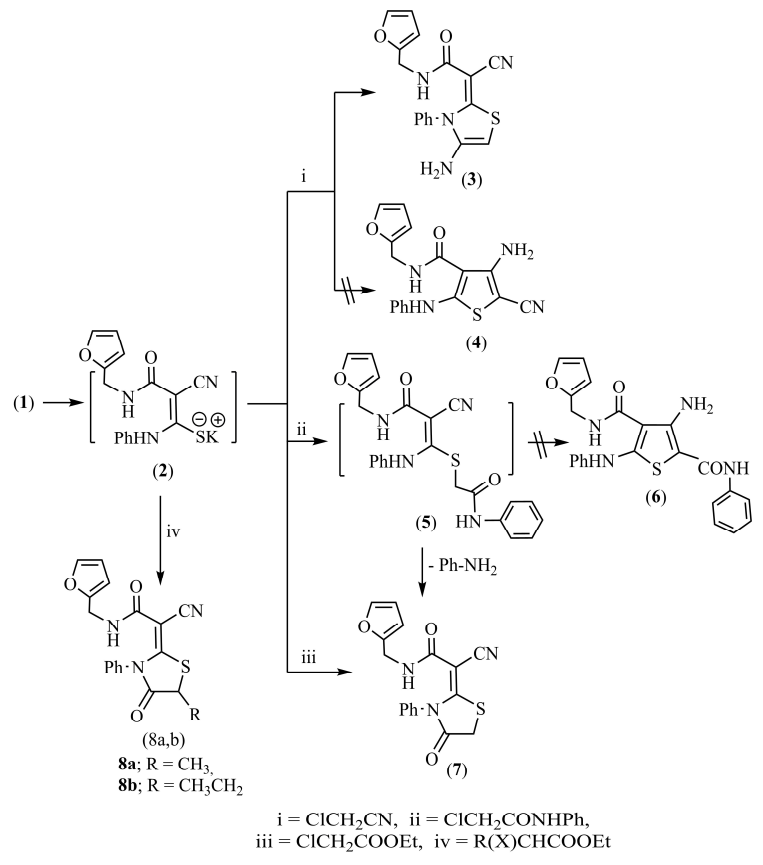

Scheme 2. Synthetic route to thiazoles.

7 was characterized by the presence of a singlet signal at $\delta$ $3.92 \mathrm{ppm}$ for thiazole methylene protons. ${ }^{13} \mathrm{C} \mathrm{NMR}$ of compound 7 revealed signals at $\delta 34.70$ ( $\mathrm{C} 5$ of thiazole), $36.32\left(\mathrm{CH}_{2}\right), 165.95,167.77$ ( $\mathrm{C} 4, \mathrm{C} 2$ of thiazole), in addition to, signals at $107.35,110.85,138.39$ and $142.37 \mathrm{ppm}$ that are characteristic for furan carbons. The structure of the isolated product $\mathbf{7}$ was elucidated also, by independent synthesis via treatment of the intermediate $\mathbf{2}$ with ethyl chloroacetate. Cycloalkylation of non-isolable salt $\mathbf{2}$ with either ethyl $\alpha$-chloropropionate or ethyl $\alpha$-bromobutyrate produced in each case, a single product. The reaction products can be formulated as thiazolidin-4-one derivatives $\mathbf{8 a}$ and $\mathbf{8 b}$, evidence for assigned structures being provided by analytical and spectroscopic data. For example, the IR spectrum of compound 8a showed the appearance of absorption band at 3326, 2203, 1733 and $1646 \mathrm{~cm}^{-1}$ corresponding to the $\mathrm{NH}, \mathrm{CN},(\mathrm{C}=\mathrm{O}$; thiazolidinone) and carboxamide groups, respectively. The ${ }^{1} \mathrm{H}$ NMR spectrum of compound 8a exhibited signals at $\delta 1.56\left(\mathrm{~d}, 3 \mathrm{H}, \mathrm{CH}_{3}\right), 4.25$ (q, $1 \mathrm{H}, \mathrm{CH}), 4.33\left(\mathrm{~d}, 2 \mathrm{H}, \mathrm{CH}_{2}\right)$ with triplet signal at $\delta 7.95$ ppm for the amide group proton. The formation of 8 was assumed to proceed via an initial alkylation followed by intramolecular cyclization with ethanol elimination (Scheme 2).

Next, treatment of the intermediate $\mathbf{2}$, formed in situ under the same reaction condition, with $\alpha$-halogenated compounds 9 produced in each case a single product identified as 4-methylthiazole derivatives $\mathbf{1 0}$ instead of the thiophene derivative $\mathbf{1 1}$ (Scheme 3). The structure of the 4methylthiazoles 10 was established by analytical and 


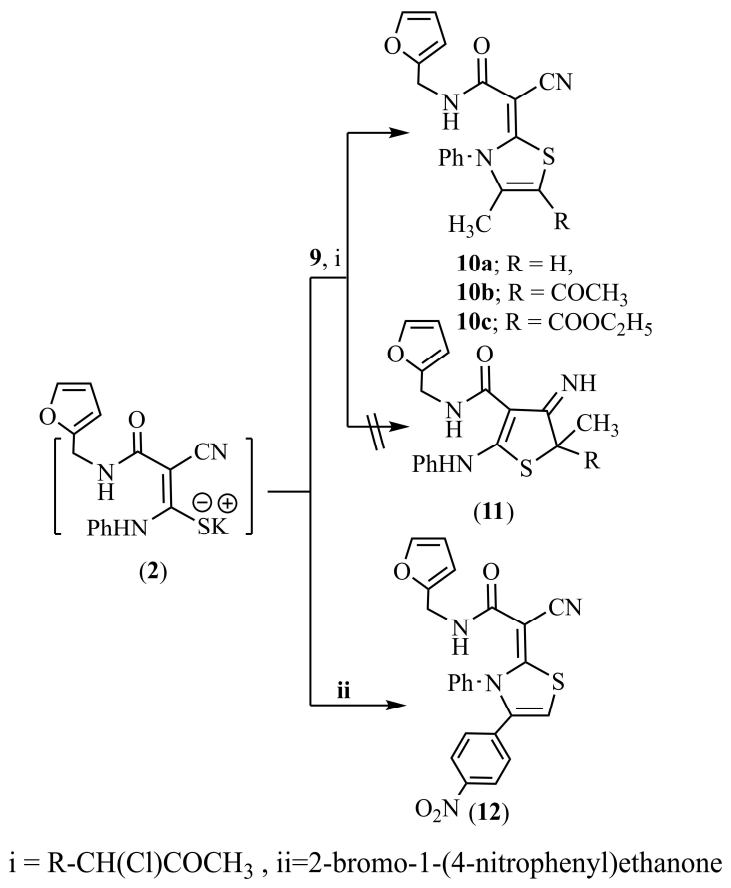

Scheme 3. Synthetic route to thiazoles.

spectroscopic data. The IR spectrum of compound 10a showed the appearance of absorption band at 3367, 2170 and $1646 \mathrm{~cm}^{-1}$ corresponding to the imino, nitrile and carboxamide groups, respectively. The ${ }^{1} \mathrm{H}$ NMR spectrum of compound 10b was characteristic by the presence of singlet signals at $\delta 2.09,2.30$ for $2 \mathrm{CH}_{3}$ with triplet signal at $\delta 9.67 \mathrm{ppm}$ for $\mathrm{NH}$ protons. ${ }^{13} \mathrm{C}$ NMR of compound $10 \mathrm{~b}$ revealed signals at $\delta 14.31,29.95\left(2 \mathrm{CH}_{3}\right), 36.10\left(\mathrm{CH}_{2}\right)$, $115.06,145.35,170.21$ (thiazole-carbons), in addition to two singlet at $\delta 163.96$ and $165.22 \mathrm{ppm}(2 \mathrm{C}=0)$. Similarly, cyclocondensation of the intermediate salt $\mathbf{2}$ with 2-bromo1-(4-nitrophenyl)ethanone at room temperature afforded the corresponding 2,3-dihydrothiazole derivative 12 (Scheme 3). The structure of the latter product was confirmed by the presence of singlet signal at $\delta 6.87 \mathrm{ppm}$ assignable for thiazole- $\mathrm{H} 5$ in ${ }^{1} \mathrm{H}$ NMR spectrum.

To investigate the structure reactivity relationship with respect to antimicrobial properties, the reactivity of 4thiazolidinone $\mathbf{7}$ toward some electrophilic reagents was investigated and bithiazolidinone derivatives were obtained. Thus, the non-isolable potassium sulphide salt $\mathbf{1 3}$ was achieved by the nucleophilic addition of active methylene group in 4-thiazolidinone $\mathbf{7}$ to phenyl isothiocyanate in dry dimethylformamide at room temperature in the presence of potassium hydroxide (Scheme 4). Cycloalkylation of non-isolable salt 13 with ethyl chloroacetate and/or ethyl $\alpha$-bromobutyrate at room temperature gave bithiazolidinone derivatives $\mathbf{1 4}$ and $\mathbf{1 5 .}$

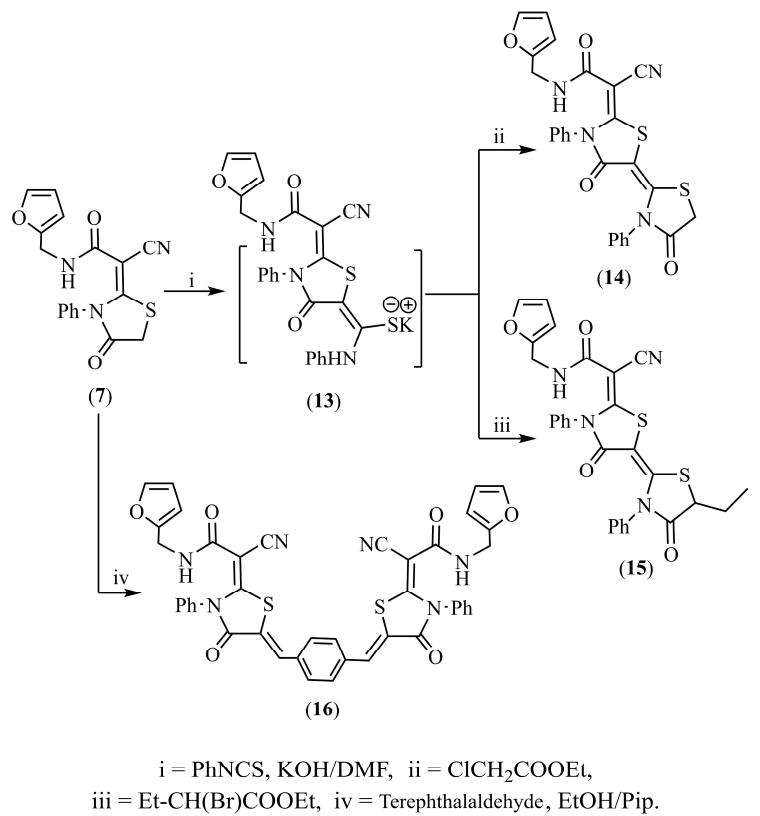

Scheme 4. Synthetic route to bithiazoles.

${ }^{13} \mathrm{C}$ NMR of compound 14 revealed signals at $\delta 31.65\left(\mathrm{CH}_{2}\right)$, 106.60 (thiazole-C5) $\left.{ }^{\prime}\right)$ 163.63, 166.12 (thiazole-C4, C4 ), in addition to singlet signal at $173.30 \mathrm{ppm} \quad(\mathrm{C}=\mathrm{O})$. Furthermore, the Knoevenagel condensation of thiazolidin4-one 7 with terephthalaldehyde in ethanolic piperidine solution under reflux conditions afforded the corresponding bithiazolidinone derivative $\mathbf{1 6}$ (Scheme 4).

In addition, pyrano[2,3- $d$ ] thiazoles are useful in the treatment of diseases such as diabetes, obesity, hyperlipidemia, and atherosclerotic diseases. ${ }^{[28]}$ They are also known to show bactericidal, fungicidal and molluscicidal activities. ${ }^{[29,30]}$ Therefore, pyrano[2,3d] thiazole of type 19 was obtained by reaction between equimolecular amounts of compound 7 and chalcone derivative $\mathbf{1 7}$ in the presence of a catalytic amount of piperidine. The structure formula of compound 19 resulting from this reaction has been confirmed using elemental analyses and spectral methods. ${ }^{1} \mathrm{H}$ NMR spectrum of compound 19 showed a new two doublet and singlet signals at $\delta 3.43,4.94,3.84$ ppm corresponding to $\mathrm{H} 4, \mathrm{H} 5$ of pyran and methoxy protons, in addition to triplet signal at $8.20 \mathrm{ppm}$ assignable for $\mathrm{NH}$ proton. The formation of $\mathbf{1 9}$ was assumed to proceed via Micheal addition of the active methylene to the $\alpha, \beta$-unsaturated ketone $\mathbf{1 7}$ to yield the Micheal adduct $\mathbf{1 8}$ followed by intramolecular cyclization with elimination of water. In contrast to the anticipated formation of pyrano[2,3-d]thiazole derivative 19, the reaction of $\mathbf{7}$ with $\alpha$-cinnamonitriles $20 a, b$ in refluxing ethanol and in the presence of a catalytic amount of piperidine, the corresponding benzyilidine thiazolidinone 


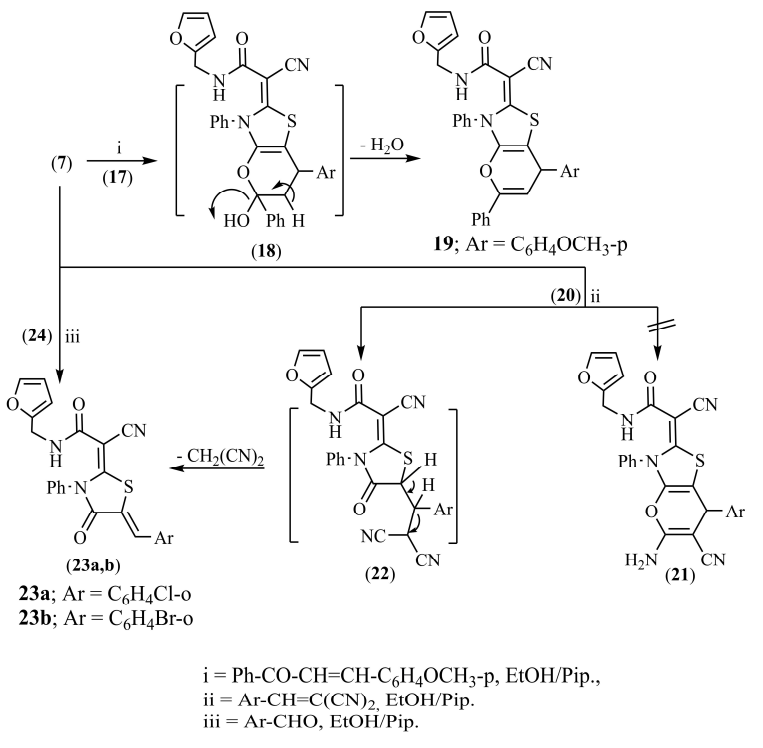

Scheme 5. Synthetic route to pyrano[2,3-d]thiazole.

derivative $\mathbf{2 3}$ was obtained. The formation of this compound was assumed to proceed via retro Michael addition through the elimination of malononitrile from the non-isolable adduct 22 . The structure of the isolated products 23a,b were elucidated on the basis of their spectral data (IR, MS and ${ }^{1} \mathrm{H} N M R$ ) and also, by independent synthesis via reaction of the thiazolidinone 7 with benzaldehyde derivatives $\mathbf{2 4}$ (Scheme 5).

\section{Antimicrobial Evaluation}

Fourteen of the newly synthesized target compounds were evaluated for their in vitro antibacterial activity against Staphylococcus aureus (RCMB 010027), Staphylococcus epidermidis (RCMB 010024) and Bacillis subitilis (RCMB 010063) as examples of Gram-positive bacteria and Pseudomonas aeruginosa (RCMB 010043), Proteous vulgaris (RCMB 010085) and Klebsiella pneumonia (RCMB 010093) as examples of Gram-negative bacteria. They were also evaluated for their in vitro antifungal potential against Aspergillus fumigatus, Aspergillus clavatus and Geotricum candidium fungal strain.

Agar-diffusion method [31] was used for the determination of the preliminary antibacterial and antifungal activity. Ampicillin, gentamycin and amphotericin B were used as reference drugs. The results were recorded for each tested compound as the average diameter of inhibition zones (IZ) of bacterial or fungal growth around the disks in $\mathrm{mm}$. The minimum inhibitory concentration (MIC) measurement was determined for compounds with significant growth inhibition zones using twofold serial dilution method. ${ }^{[32]}$ The MIC $(\mu \mathrm{g} / \mathrm{mL})$ and inhibition zone diameters values are presented in Table 1 . The results depicted in Table 1 revealed that most of tested compounds displayed variable inhibitory effects on the growth of the tested Gram-positive and Gram-negative bacterial strains and also against antifungal strains.

Regarding the structure reactivity relationship between the thiazoles and antibacterial activities, the

Table 1. Antimicrobial inhibition zone in $\mathrm{mm}$ and minimal inhibitory concentrations ( $\mathrm{MIC}, \mu \mathrm{g} / \mathrm{ml}$, between brackets) of some new synthesized compounds

\begin{tabular}{|c|c|c|c|c|c|c|c|c|c|}
\hline \multirow[t]{2}{*}{ Compounds no. } & \multicolumn{3}{|c|}{ Gram-positive bacteria } & \multicolumn{3}{|c|}{ Gram-negative bacteria } & \multicolumn{3}{|c|}{ Fungi } \\
\hline & S. aureus & S. epidermidis & B. subitilis & $P$. aeruginosa & P. vulgaris & K. pneumonia & A. fumigatus & A. clavatus & G. candidium \\
\hline 3 & 14.3 & 16.2 & 16.2 & $N A^{*}$ & 14.2 & 14.8 & 14.6 & 14.9 & 15.7 \\
\hline 7 & 15.3 & 17.4 & 17.9 & NA & 13.6 & 15.4 & 12.3 & 14.2 & 14.9 \\
\hline $8 a$ & 19.6 & 20.1 & 21.2 & NA & 17.6 & 19.1 & 17.2 & 19.6 & 20.9 \\
\hline $8 b$ & 18.1 & 20.2 & 20.9 & NA & 16.2 & 19.9 & 16.3 & 18.2 & 20.3 \\
\hline $10 a$ & 20.6 & 22.3 & 20.7 & NA & 16.3 & 18.1 & 16.3 & 19.2 & 19.6 \\
\hline $10 \mathrm{~b}$ & 20.6 & 22.3 & 23.1 & NA & 21.2 & 23.6 & 18.6 & 19.2 & 20.9 \\
\hline $10 c$ & 17.4 & 17.9 & 19.2 & NA & 16.3 & 17.2 & 13.3 & 15.2 & 17.3 \\
\hline 12 & $20.2(3.9)$ & $21.4(1.95)$ & $23.6(0.98)$ & NA & $18.3(7.81)$ & $22.4(0.98)$ & $18.2(7.81)$ & $21.4(1.95)$ & $23.2(0.98)$ \\
\hline 14 & $21.2(1.95)$ & $21.9(0.98)$ & $23.7(0.48)$ & NA & $19.2(3.9)$ & $23.3(0.98)$ & $20.3(3.9)$ & $22.3(0.98)$ & $23.6(0.49)$ \\
\hline 15 & $21.8(1.95)$ & $22.7(0.98)$ & $24.1(0.48)$ & NA & $20.1(3.9)$ & $23.1(0.98)$ & 19.7(3.9) & $22.7(0.98)$ & $23.8(0.49)$ \\
\hline 16 & $20.3(3.9)$ & $21.2(1.95)$ & $22.9(0.98)$ & NA & $20.3(3.9)$ & $22.4(0.98)$ & $20.1(3.9)$ & $21.9(0.98)$ & $23.5(0.98)$ \\
\hline 19 & 16.2 & 17.5 & 18.1 & NA & 14.2 & 15.7 & 14.3 & 16.2 & 15.4 \\
\hline $23 a$ & $21.4(1.95)$ & $22.3(0.98)$ & $23.6(0.49)$ & NA & $21.4(1.95)$ & $23.6(0.49)$ & $22.3(0.98)$ & $22.7(0.98)$ & $23.9(0.98)$ \\
\hline $23 b$ & $20.3(1.93)$ & $19.2(0.98)$ & $22.4(0.49)$ & NA & $19.3(1.95)$ & $21.3(0.49)$ & $21.1(0.98)$ & $20.7(0.98)$ & $22.9(0.98)$ \\
\hline Ampicillin & $28.9(0.24)$ & $25.4(0.49)$ & $36.6(0.24)$ & $N A^{*}$ & NT & NT & NT & NT & NT \\
\hline Gentamycin & $N T^{*}$ & NT & NT & 19.9(3.9) & $25.4(0.98)$ & $26.3(0.49)$ & NT & NT & NT \\
\hline Amphotericin B & NT & NT & NT & NT & NT & NT & $23.7(0.98)$ & $21.9(0.98)$ & $25.4(0.49)$ \\
\hline
\end{tabular}

*NA: no activity, ${ }^{*}$ NT: not tested 


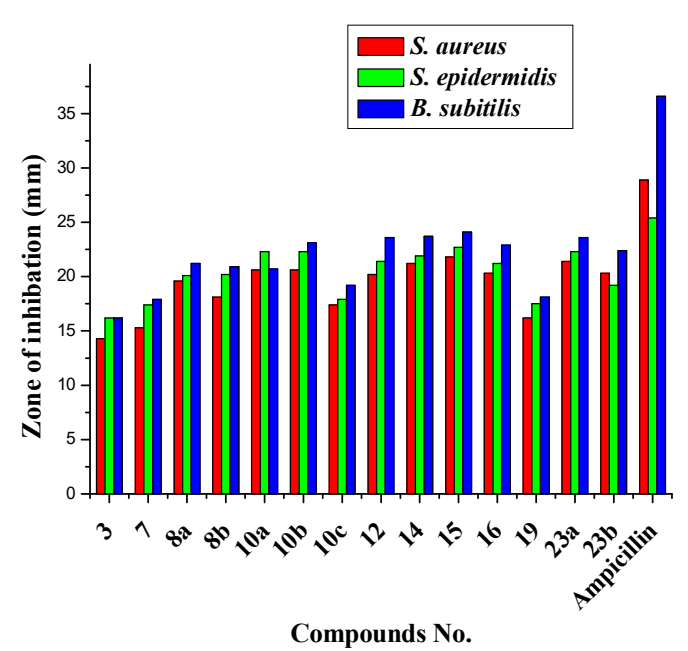

Figure 1. Antibacterial activity of the synthesized compounds against Gram-positive bacteria.

results showed that thiazole benzyilidine derivatives 23a, 23b and bithiazolodinone derivatives 14-16 were the most active among the synthesized compounds (Figures 1 and 2). Thiazoles 10b, 10c and 12 containing electron withdrawing groups such as $\mathrm{COCH}_{3}, \mathrm{CO}_{2} \mathrm{C}_{2} \mathrm{H}_{5}$ and $\mathrm{NO}_{2}$ recorded moderate to good activity. In this view, compounds 23a and 23b were equipotent to gentamycin against $K$. pneumonia (MIC $0.49 \mu \mathrm{g} / \mathrm{mL}$ ), while their activity were $50 \%$ lower than both gentamycin and ampicillin in inhibiting the growth of P. vulgaris (MIC $1.95 \mu \mathrm{g} / \mathrm{mL}$ ), S. epidermidis (MIC 0.98 $\mu \mathrm{g} / \mathrm{mL}$ ) and $B$. subitilis (MIC $0.49 \mu \mathrm{g} / \mathrm{mL}$ ). Bithiazolodinone derivatives 14 and 15 showed $50 \%$ of the activity of ampicillin and gentamycin against S. epidermidis (MIC 0.98 $\mu \mathrm{g} / \mathrm{mL}$ ), B. subitilis (MIC $0.49 \mu \mathrm{g} / \mathrm{mL}$ ). Bithiazolodinone 20 showed relatively good growth inhibitory profiles against $K$. pneumonia (MIC $0.98 \mu \mathrm{g} / \mathrm{mL}$ ) which was about $50 \%$ of the activity of gentamycin. The 4-nitrophenylthiazole $\mathbf{1 2}$ showed relatively good growth inhibitory profiles against $B$. subitilis (MIC $0.98 \mu \mathrm{g} / \mathrm{mL}$ ) and $K$. pneumonia (MIC 0.98 $\mu \mathrm{g} / \mathrm{mL}$ ), which was about $50 \%$ of the activity of ampicillin and gentamycin. Concerning the antibacterial activity of the compounds $3,7,8 a, 8 b, 10 a, 10 c$ and 19 showed weak activities against the tested Gram-positive and Gramnegative bacteria. Unfortunately, all the tested compounds were completely inactive toward $P$. aeruginosa (RCMB 010043) compared to gentamycin. Gram negative bacteria have a relatively thin cell wall constituted by few layers of peptidoglycan surrounded by a second lipid membrane containing lipopolysaccharides and lipoproteins. These differences in cell wall structure can produce differences in antibacterial susceptibility and some antibiotics are ineffective against Gram-negative pathogens. ${ }^{[33-35]}$

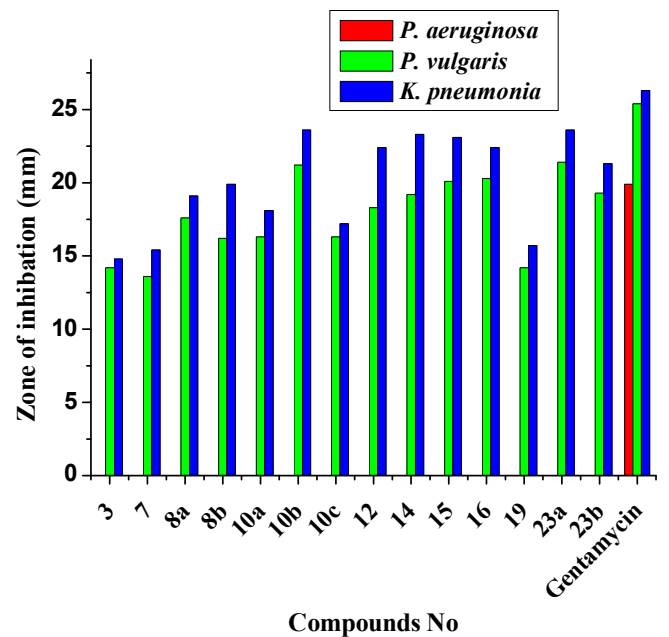

Figure 2. Antibacterial activity of the synthesized compounds against Gram-negative bacteria.

Regarding the activity of thiazoles incorporating furan moiety versus antifungal strains, thiazole benzyildine 23a and 23b showed in vitro antifungal activity equipotent to amphotericin B against $A$. fumigatus (MIC $0.98 \mu \mathrm{g} / \mathrm{mL}$ ), A. clavatus (MIC $0.98 \mu \mathrm{g} / \mathrm{mL}$ ) and G. candidium (MIC 0.49 $\mu \mathrm{g} / \mathrm{mL}$ ). Bithiazolidinones $\mathbf{1 4}$ and $\mathbf{1 5}$ were equipotent to amphote-ricin B in inhibiting the growth of $A$. clavatus (MIC $0.98 \mu \mathrm{g} / \mathrm{mL}$ ) and G. candidium (MIC $0.49 \mu \mathrm{g} / \mathrm{mL}$ ). But their activities were $25 \%$ of amphote-ricin B against $A$. fumigatus (MIC $3.9 \mu \mathrm{g} / \mathrm{mL}$ ). indeed, Bithiazolidinones 16 displayed equipotent activity to amphotericin B versus $A$. clavatus (MIC $0.98 \mu \mathrm{g} / \mathrm{mL}$ ) while its activity was $50 \%$ lower than $G$. candidium (MIC $0.98 \mu \mathrm{g} / \mathrm{mL}$ ) compared to amphotericin B. 4-Nitrophenylthiazole 12 showed relatively good growth inhibitory profiles versus A. clavatus (MIC $1.95 \mu \mathrm{g} / \mathrm{mL}$ ) and G. candidium (MIC $0.98 \mu \mathrm{g} / \mathrm{mL}$ ) which was about $50 \%$ of the activity of amphotericin B. Other synthesized compounds showed weak to moderate activity against antifungal strains (Figure 3).

\section{CONCLUSION}

The objective of the present study was to synthesize and investigate the antimicrobial activities of some new thiazoles incorporating furan moiety with the hope of discovering new structure leads serving as potent antimicrobial agents. Our aim has been verified by the synthesis of thiazole, bithiazoles, thiazole benzyilidine and pyranothiazole derivatives. It is worth mentioning that, synthesis of thiazole derivative containing electron withdrawing groups such as $\mathbf{1 0 b}, \mathbf{1 0 c}$ and $\mathbf{1 2}$ exhibited good antimicrobial activity. The best antimicrobial activity was 


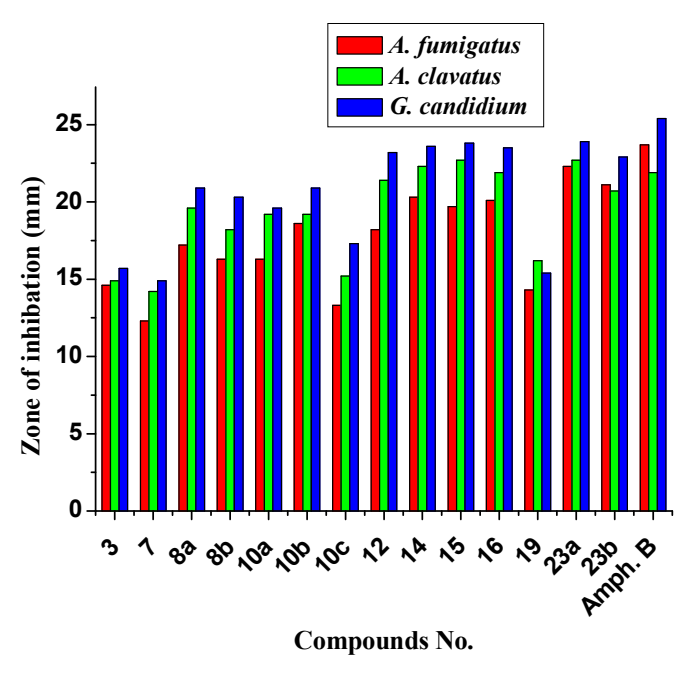

Figure 3. Antifungal activity of the synthesized compounds.

observed when 4-thiazoledinone 7 was converted into bithiazoles 14-16 or thiazole benzyilidine derivatives 23a, 23b.

\section{EXPERIMENTAL}

All the melting points are uncorrected. IR $(\mathrm{KBr})$ spectra were measured on Shimadzu 440 spectrometer; ${ }^{1 H}$ NMR and ${ }^{13} \mathrm{C}$ NMR spectra were obtained in DMSO on a Varian Gemini $300 \mathrm{MHz}$ spectrometer using TMS as internal standard. The chemical shifts are reported as $\delta_{\mathrm{ppm}}$ units. Mass spectra were obtained on GCMS $\backslash Q P 1000$ Ex mass spectrometer at $70 \mathrm{ev}$. Elemental analyses were carried out at the Department of Chemistry, Faculty of Science, Cairo University, Egypt. Microbiology screening was carried out in the Regional Center for Microbiology and Biotechnology (RCMB), Antimicrobial unit test organisms, Al-azhr University, Cairo, Egypt.

\section{General procedure for the preparation of compounds $3,7,8 a, b, 10 a-c, 12,14$ and 15:}

To suspension of finely powdered potassium hydroxide $(0.01 \mathrm{~mol})$ in dry dimethylformamide $(20 \mathrm{~mL})$ the active methylene compound ( 1 and/or 7, $0.01 \mathrm{~mol}$ ) and then the phenyl isothiocyanate $(0.01 \mathrm{~mol})$ were added in portions. The reaction mixture was stirred at room temperature for $1 \mathrm{~h}$ and then treated with $\alpha$-halogenated compound $(0.01$ mol) and left at room temperature for $2 \mathrm{~h}$; then it was poured into ice/water and acidified with $0.1 \mathrm{M} \mathrm{HCl}$ at pH 34. The resulting precipitate was filtered off, dried, and recrystallized from the proper solvent.

\section{2-(4-Amino-3-phenylthiazol-2(3H)-ylidene)-2-cyano- $\mathrm{N}$ - (furan-2-ylmethyl)acetamide (3)}

Brown crystals (AcOH), Yield (50\%), m.p. 229-230ㄷ; IR (KBr) $v_{\max } / \mathrm{cm}^{-1}: 3423,3308,3110\left(\mathrm{NH}_{2} / \mathrm{NH}\right), 3058$ (arom. $\mathrm{CH}), 2184(\mathrm{C} \equiv \mathrm{N})$ and 1637 (C=O; amide); ${ }^{1} \mathrm{H}$ NMR (DMSO$\left.d_{6}\right) \delta / p p m: 4.27\left(\mathrm{~d}, 2 \mathrm{H}, \mathrm{C}_{2}-\mathrm{NH}, J=6 \mathrm{~Hz}\right), 6.14(\mathrm{~d}, 1 \mathrm{H}$, furan$\mathrm{H} 3, J=3 \mathrm{~Hz}$ ), $6.34(\mathrm{t}, \mathrm{H}$, furan-H4, $J=3 \mathrm{~Hz}), 7.16(\mathrm{~s}, 1 \mathrm{H}$, thiazole-H5), 7.30-7.60 (m, 6H, Ar-H + furan-H5), $8.22(\mathrm{~s}$, $\left.2 \mathrm{H}, \mathrm{NH}_{2}\right), 10.08$ (t, $\left.1 \mathrm{H}, \mathrm{O}=\mathrm{C}-\mathrm{NH}-\mathrm{CH}_{2}, J=6 \mathrm{~Hz}\right) . \mathrm{MS}(\mathrm{El}, 70 \mathrm{ev})$ $\mathrm{m} / \mathrm{z}$ (\%): 338 (M+, 55), 309 (15.2), 242 (35), 215 (39.7), 135 (72.5), 64 (100) 51 (34.3). Anal. Calcd. for $\mathrm{C}_{17} \mathrm{H}_{14} \mathrm{~N}_{4} \mathrm{O}_{2} \mathrm{~S}$ (338.38): C, 60.34; H, 4.17; N, 16.56. Found: C, 60.14; H, $4.20 ; \mathrm{N}, 16.39 \%$.

2-Cyano-N-(furan-2-ylmethyl)-2-(4-oxo-3phenylthiazolidin-2-ylidene)acetamide (7)

White crystals (EtOH), Yield (80\%), m.p. 108-110 ㄷ; IR $(\mathrm{KBr}) v_{\max } / \mathrm{cm}^{-1}: 3339(\mathrm{NH}), 3036$ (arom. $\left.\mathrm{CH}\right), 2206(\mathrm{C}=\mathrm{N})$, 1719 (C=O; thiazoldinone) and 1645 ( $\mathrm{C}=\mathrm{O}$; amide); ${ }^{1 \mathrm{H}} \mathrm{NMR}$

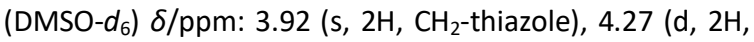
$\left.\mathrm{CH}_{2}-\mathrm{NH}, J=6 \mathrm{~Hz}\right), 6.14(\mathrm{~d}, 1 \mathrm{H}$, furan-H3, $J=3 \mathrm{~Hz}), 6.36(\mathrm{t}, \mathrm{H}$, furan- $\mathrm{H} 4, \mathrm{~J}=3 \mathrm{~Hz}$ ), 7.23-8.17 (m, 6H, Ar-H + furan-H5), 8.17 (t, $1 \mathrm{H}, \mathrm{O}=\mathrm{C}-\mathrm{NH}-\mathrm{CH}_{2}, J=6 \mathrm{~Hz}$ ); ${ }^{13} \mathrm{C}$ NMR (DMSO- $d_{6}$ ) $\delta / \mathrm{ppm}$ : 34.70 (thiazole-C5), $36.32\left(\mathrm{CH}_{2}\right), 80.90(\underline{\mathrm{C}}-\mathrm{CN}), 107.35$, $110.58,138.39,142.37$ (furan-C), 124.40, 127.0, 128.20, 129.72, 129.87 (Ar-C), 152.63 (CN), 165.95, 167.77 (thiazole-C4, C2), 173.85 (C=O). MS (El, 70 ev) m/z (\%): 339 $\left(\mathrm{M}^{+}, 70\right), 310$ (9.2), 298 (18), 265 (27), 236 (22.3), 96 (37.8), 81 (100) 53 (38.3). Anal. Calcd. for $\mathrm{C}_{17} \mathrm{H}_{13} \mathrm{~N}_{3} \mathrm{O}_{3} \mathrm{~S}$ (339.37): C, $60.17 ; \mathrm{H}, 3.86 ; \mathrm{N}, 12.38$. Found: $\mathrm{C}, 60.31 ; \mathrm{H}, 3.66 ; \mathrm{N}, 12.20 \%$.

\section{2-Cyano- $\mathrm{N}$-(furan-2-ylmethyl)-2-(5-methyl-4-oxo-3-} phenylthiazolidin-2-ylidene)acetamide (8a)

White crystals (AcOH), Yield (60\%), m.p. 190-192 oC; IR $(\mathrm{KBr}) v \mathrm{max} / \mathrm{cm}^{-1}$ : $3326(\mathrm{NH}), 3060$ (arom. $\mathrm{CH}$ ), 2915 (aliph. $\mathrm{CH}), 2203(\mathrm{C} \equiv \mathrm{N}), 1733(\mathrm{C}=\mathrm{O}$; thiazoldinone) and $1646(\mathrm{C}=\mathrm{O}$; amide); ${ }^{1} \mathrm{H}$ NMR (DMSO- $\left.d_{6}\right) \delta /$ ppm: $1.56\left(\mathrm{~d}, 3 \mathrm{H}, \mathrm{CH}_{3} J=9\right.$ $\mathrm{Hz}$ ), 4.25 (q, $1 \mathrm{H}, \mathrm{CH}, J=9 \mathrm{~Hz}), 4.33\left(\mathrm{~d}, 2 \mathrm{H}, \mathrm{CH}_{2}-\mathrm{NH}, J=6 \mathrm{~Hz}\right)$, $6.16(\mathrm{~d}, 1 \mathrm{H}$, furan- $\mathrm{H} 3, J=3 \mathrm{~Hz}), 6.35(\mathrm{t}, 1 \mathrm{H}$, furan- $\mathrm{H} 4, J=3$ $\mathrm{Hz}), 7.38-7.51(\mathrm{~m}, 6 \mathrm{H}, \mathrm{Ar}-\mathrm{H}+$ furan-H5), $7.95(\mathrm{t}, 1 \mathrm{H}, \mathrm{O}=\mathrm{C}-$ $\left.\mathrm{NH}-\mathrm{CH}_{2}, J=6 \mathrm{~Hz}\right) . \mathrm{MS}(\mathrm{El}, 70 \mathrm{ev}) \mathrm{m} / \mathrm{z}(\%): 353\left(\mathrm{M}^{+}, 67\right), 248$ (33.2), 229 (58.5), 203 (17), 142 (22.3), 77(100) 53 (65.3). Anal. Calcd. for $\mathrm{C}_{18} \mathrm{H}_{15} \mathrm{~N}_{3} \mathrm{O}_{3} \mathrm{~S}$ (353.39): $\mathrm{C}, 61.18 ; \mathrm{H}, 4.28 ; \mathrm{N}$, 11.89. Found: C, $60.98 ; \mathrm{H}, 4.15 ; \mathrm{N}, 11.72 \%$.

\section{2-Cyano-2-(5-ethyl-4-oxo-3-phenylthiazolidin-2-ylidene)- $\mathrm{N}$-(furan-2-ylmethyl)acetamide (8b)}

White crystals (AcOH), Yield (55\%), m.p. 199-200 ㅇ; IR $(\mathrm{KBr}) v_{\max } / \mathrm{cm}^{-1}$ : $3321(\mathrm{NH}), 3040$ (arom. $\mathrm{CH}$ ), 2916 (aliph. $\mathrm{CH}), 2203(\mathrm{C} \equiv \mathrm{N}), 1732$ ( $\mathrm{C}=\mathrm{O}$; thiazoldinone) and $1646(\mathrm{C}=\mathrm{O}$; amide); ${ }^{1} \mathrm{H}$ NMR (DMSO- $d_{6}$ ) $\delta /$ ppm: $1.03\left(\mathrm{t}, 3 \mathrm{H}, \mathrm{CH}_{3}, J=6\right.$ $\mathrm{Hz}), 1.96\left(\mathrm{p}, 2 \mathrm{H}, \mathrm{CH}_{2}, J=6 \mathrm{~Hz}\right), 4.19(\mathrm{t}, 1 \mathrm{H}, \mathrm{CH}, J=6 \mathrm{~Hz}), 4.27$ 
(d, $2 \mathrm{H}, \mathrm{CH}_{2}-\mathrm{NH}, J=6 \mathrm{~Hz}$ ), 6.17 (d, $1 \mathrm{H}$, furan- $\mathrm{H3}, J=3 \mathrm{~Hz}$ ), $6.35(\mathrm{t}, 1 \mathrm{H}$, furan- $\mathrm{H} 4, J=3 \mathrm{~Hz}), 7.35-7.51(\mathrm{~m}, 6 \mathrm{H}, \mathrm{Ar}-\mathrm{H}+$ furan-H5), 7.94 (t, $\left.1 \mathrm{H}, \mathrm{O}=\mathrm{C}-\mathrm{N} \underline{\mathrm{H}}-\mathrm{CH}_{2}, J=6 \mathrm{~Hz}\right) . \mathrm{MS}(\mathrm{El}, 70 \mathrm{ev})$ m/z (\%): 367( $\left.\mathrm{M}^{+}, 87\right), 338$ (7), 271 (37.2), 265 (5.5), 243 (21.5), 217 (12), 96 (50.3), 80 (100), 64 (82), 53 (25). Anal. Calcd. for $\mathrm{C}_{19} \mathrm{H}_{17} \mathrm{~N}_{3} \mathrm{O}_{3} \mathrm{~S}$ (367.42): C, 62.11; $\mathrm{H}, 4.66 ; \mathrm{N}, 11.44$. Found: $\mathrm{C}, 61.90 ; \mathrm{H}, 4.46 ; \mathrm{N}, 11.60 \%$.

\section{2-Cyano-N-(furan-2-ylmethyl)-2-(4-methyl-3-} phenylthiazol-2(3H)-ylidene)acetamide (10a)

White powder (EtOH), Yield (70\%), m.p. 240-241 ㅇ; IR $(\mathrm{KBr}) v_{\max } / \mathrm{cm}^{-1}: 3376(\mathrm{NH}), 3046$ (arom. $\mathrm{CH}$ ), 2926 (aliph. $\mathrm{CH}$ ), $2170\left(\mathrm{C} \equiv \mathrm{N}\right.$ ) and 1646 ( $\mathrm{C}=\mathrm{O}$; amide); ${ }^{1} \mathrm{H}$ NMR (DMSO- $d_{6}$ ) $\delta / p p m: 1.80\left(\mathrm{~s}, 3 \mathrm{H}, \mathrm{CH}_{3}\right), 4.29\left(\mathrm{~d}, 2 \mathrm{H}, \mathrm{CH}_{2}-\mathrm{NH}, J=6 \mathrm{~Hz}\right), 6.12$ (d, $1 \mathrm{H}$, furan- $\mathrm{H} 3, J=3 \mathrm{~Hz}), 6.33(\mathrm{t}, 1 \mathrm{H}$, furan- $\mathrm{H} 4, J=3 \mathrm{~Hz}$ ), $6.80(\mathrm{~s}, 1 \mathrm{H}$, thiazole-H5), 7.12-7.56 (m, 7H, Ar-H + furan-H5

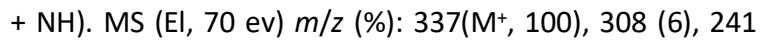
(78.5), 214 (45), 189 (57), 118 (30.3), 96(18), 77 (64), 53 (78). Anal. Calcd. for $\mathrm{C}_{18} \mathrm{H}_{15} \mathrm{~N}_{3} \mathrm{O}_{2} \mathrm{~S}$ (337.40): C, 64.08; $\mathrm{H}$, 4.48; N, 12.45. Found: $C, 64.00 ; \mathrm{H}, 4.55 ; \mathrm{N}, 12.33 \%$.

\section{2-(5-Acetyl-4-methyl-3-phenylthiazol-2(3H)-ylidene)-2- cyano- $\mathbf{N}$-(furan-2-ylmethyl)acetamide (10b)}

White powder (AcOH), Yield (66 \%), m.p. 221-222 ㅇ; IR (KBr) $v_{\max } / \mathrm{cm}^{-1}$ : $3316(\mathrm{NH}), 3061$ (arom. CH), 2900 (aliph. $\mathrm{CH}), 2190(\mathrm{C} \equiv \mathrm{N}), 1699(\mathrm{C}=\mathrm{O})$ and $1617\left(\mathrm{C}=\mathrm{O}\right.$; amide); ${ }^{1} \mathrm{H}$ NMR (DMSO- $\left.d_{6}\right): \delta / p p m: 2.09\left(\mathrm{~s}, 3 \mathrm{H}, \mathrm{CH}_{3}\right), 2.30(\mathrm{~s}, 3 \mathrm{H}$, $\left.\mathrm{COCH}_{3}\right), 4.29\left(\mathrm{~d}, 2 \mathrm{H}, \mathrm{C}_{2}-\mathrm{NH}, J=6 \mathrm{~Hz}\right), 6.15(\mathrm{~d}, 1 \mathrm{H}$, furan-H3, $J=3 \mathrm{~Hz}), 6.34(\mathrm{t}, 1 \mathrm{H}$, furan- $\mathrm{H} 4, J=3 \mathrm{~Hz}), 7.45-7.66(\mathrm{~m}, 6 \mathrm{H}$, $\mathrm{Ar}-\mathrm{H}+$ furan- $\mathrm{H} 5), 9.67\left(\mathrm{t}, 1 \mathrm{H}, \mathrm{O}=\mathrm{C}-\mathrm{NH}-\mathrm{CH}_{2}, \mathrm{~J}=6 \mathrm{~Hz}\right) ;{ }^{13} \mathrm{CNMR}$ (DMSO-d $\left.)_{6}\right) \delta / p p m: 14.31\left(\mathrm{CH}_{3}\right), 29.95\left(\mathrm{COCH}_{3}\right), 36.10\left(\mathrm{CH}_{2}\right)$, 68.83 (C-CN), 103.52, 110.32, 141.59, 141.74 (furan-C), 115.06, 145.35, 170.21 (thiazole-C), 128.90, 129.30, 129.77, 131.23, 135.97 (ph-C), 152.80 (CN), 163.96, 165.22 (2C=O). MS (El, 70 ev) m/z (\%): $379\left(\mathrm{M}^{+}, 35\right), 350$ (7), 283 (12.2), 265 (9), 241 (7.5), 231 (15), 142 (4.3), 80 (100), 64 (85), 53 (10). Anal. Calcd. for $\mathrm{C}_{20} \mathrm{H}_{17} \mathrm{~N}_{3} \mathrm{O}_{3} \mathrm{~S}$ (379.43). C, 63.31; $\mathrm{H}, 4.52 ; \mathrm{N}$, 11.07. Found: $\mathrm{C}, 63.20 ; \mathrm{H}, 4.45 ; \mathrm{N}, 10.95 \%$.

Ethyl 2-(1-cyano-2-((furan-2-ylmethyl)amino)-2oxoethylidene)-4-methyl-3-phenyl-2,3-dihydrothiazole-5carboxylate (10c)

White crystals (Dioxane), Yield (55\%), m.p. 231-232 ㄷ; IR $(\mathrm{KBr}) v_{\max } / \mathrm{cm}^{-1}: 3410(\mathrm{NH}), 3066$ (arom. $\mathrm{CH}$ ), 2920 (aliph. $\mathrm{CH}), 2183(\mathrm{C} \equiv \mathrm{N}), 1703$ ( $\mathrm{C}=\mathrm{O}$; ester) and 1633 ( $\mathrm{C}=\mathrm{O}$; amide); ${ }^{1} \mathrm{H}$ NMR (DMSO- $d_{6}$ ) $\delta / p p m: 1.29\left(\mathrm{t}, 3 \mathrm{H}, \mathrm{CH}_{3}, J=6 \mathrm{~Hz}\right.$ ), 2.12 (s, $\left.3 \mathrm{H}, \mathrm{CH}_{3}\right), 3.45\left(\mathrm{q}, 2 \mathrm{H}, \mathrm{CH}_{2}, J=6 \mathrm{~Hz}\right), 4.30\left(\mathrm{~d}, 2 \mathrm{H}, \mathrm{CH}_{2}-\mathrm{NH}\right.$, $J=6 \mathrm{~Hz}), 6.15(\mathrm{~d}, 1 \mathrm{H}$, furan- $\mathrm{H} 3, J=3.3 \mathrm{~Hz}), 6.35(\mathrm{t}, 1 \mathrm{H}$, furan$\mathrm{H} 4, J=3.5 \mathrm{~Hz}$ ), 7.44-7.62 (m, 7H, Ar-H + furan-H5 + NH). MS (El, 70 ev) m/z (\%): 409 (M+, 100), 380 (7), 285 (47.5), 261 (57), 212 (15), 169 (15.3), 118 (22), 77 (88), 53 (78). Anal. Calcd. for $\mathrm{C}_{21} \mathrm{H}_{19} \mathrm{~N}_{3} \mathrm{O}_{4} \mathrm{~S}$ (409.46): C, 61.60; $\mathrm{H}, 4.68 ; \mathrm{N}, 10.26$. Found: C, $61.50 ; \mathrm{H}, 4.57$; N, $10.17 \%$.

\section{2-Cyano-N-(furan-2-ylmethyl)-2-(4-(4-nitrophenyl)-3-} phenylthiazol-2(3H)ylidene)acetamide (12)

Yellow powder (EtOH), Yield (60\%), m.p. 210-211 ㄷ; IR $(\mathrm{KBr}) v_{\max } / \mathrm{cm}^{-1}$ : $3336(\mathrm{NH}), 3080$ (arom. CH), 2934 (aliph. $\mathrm{CH}), 2189\left(\mathrm{C} \equiv \mathrm{N}\right.$ ) and 1637 (C=O; amide); ${ }^{1} \mathrm{H}$ NMR (DMSO- $d_{6}$ ) $\delta / p p m: 4.32\left(\mathrm{~d}, 2 \mathrm{H}, \underline{\mathrm{C}}_{2}-\mathrm{NH}, J=6 \mathrm{~Hz}\right), 6.15(\mathrm{~d}, 1 \mathrm{H}$, furan- $\mathrm{H} 3$, $J=3.5 \mathrm{~Hz}), 6.35(\mathrm{t}, 1 \mathrm{H}$, furan-H4, $J=3 \mathrm{~Hz}), 6.87(\mathrm{~s}, 1 \mathrm{H}$, thiazole-H5), 7.09-8.19 (m, 11H, Ar-H + furan-H5 + NH). MS (El, 70 ev) $\mathrm{m} / \mathrm{z}(\%):$ 444( $\left.\mathrm{M}^{+}, 48\right), 364$ (60), 321 (52.5), 302 (37), 274 (39), 134 (5.3), 89 (15), 80 (82), 64 (100). Anal. Calcd. for $\mathrm{C}_{23} \mathrm{H}_{16} \mathrm{~N}_{4} \mathrm{O}_{4} \mathrm{~S}$ (444.46): C, 62.15; H, 3.63; N, 12.61 . Found: $\mathrm{C}, 62.04 ; \mathrm{H}, 3.80 ; \mathrm{N}, 12.51$.

\section{2-Cyano-2-(4,4'-dioxo-3,3'-diphenyl-3',4,4',5-tetrahydro- 2'H,3H-[2,5'-bi-thiazolylidene]-2'-ylidene)-N-(furan-2- ylmethyl)acetamide (14)}

Beige crystals (AcOH), Yield (45 \%), m.p. 285-286 ㄷ; IR (KBr) $v_{\max } / \mathrm{cm}^{-1}: 3373(\mathrm{NH}), 3054$ (arom. $\mathrm{CH}$ ), 2932 (aliph. $\mathrm{CH}$ ), $2190(\mathrm{C} \equiv \mathrm{N}), 1730(\mathrm{C}=\mathrm{O}$; thiazoldinone $)$ and $1646(\mathrm{C}=\mathrm{O}$;

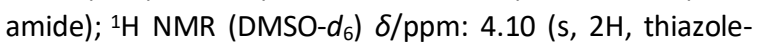
$\left.\mathrm{CH}_{2}\right), 4.17\left(\mathrm{~d}, 2 \mathrm{H}, \mathrm{CH}_{2}-\mathrm{NH}, J=6 \mathrm{~Hz}\right), 6.08(\mathrm{~d}, 1 \mathrm{H}$, furan- $\mathrm{H} 3, J$ $=3.5 \mathrm{~Hz}), 6.33(\mathrm{t}, 1 \mathrm{H}$, furan- $\mathrm{H} 4, \mathrm{~J}=3 \mathrm{~Hz}), 7.26-8.18(\mathrm{~m}, 11 \mathrm{H}$, Ar-H + furan-H5), $9.75\left(\mathrm{t}, 1 \mathrm{H}, \mathrm{O}=\mathrm{C}-\mathrm{N} \underline{\mathrm{H}}-\mathrm{CH}_{2}, J=6 \mathrm{~Hz}\right) ;{ }^{13} \mathrm{CNMR}$

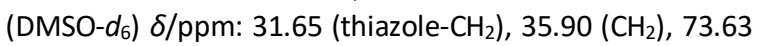
(C-CN), 106.60 (thiazole-C5'), 110.35, 152.24, 153.23 (furanC), 113.90 (CN), 129.81, 129.92, 130.32, 133.86, 134.93 (ArC), 161.99 (thiazole-C2), 163.63, 166.12 (thiazole-C4, C4'), 173.30 (C=O). MS (El, $70 \mathrm{ev}) \mathrm{m} / z(\%): 514\left(\mathrm{M}^{+}, 36\right), 434$ (50.2), 391 (21.5), 221 (78), 197 (68), 103 (43), 77 (100), 64 (40). Anal. Calcd. for $\mathrm{C}_{26} \mathrm{H}_{18} \mathrm{~N}_{4} \mathrm{O}_{4} \mathrm{~S}_{2}$ (514.58): $\mathrm{C}, 60.69 ; \mathrm{H}, 3.53 ; \mathrm{N}$, 10.89. Found: C, 60.49; H, 3.40; N, $10.95 \%$.

2-Cyano-2-(5-ethyl-4,4'-dioxo-3,3'-diphenyl-3',4,4',5tetrahydro-2' $\mathrm{H},-3 \mathrm{H}-\left[2,5^{\prime}\right.$-bithiazolylidene]-2'-ylidene)- $\mathrm{N}$ (furan-2-ylmethyl)acetamide(15)

Beige crystals (AcOH), Yield (45\%), m.p. 279-280 C; IR (KBr) $v_{\text {max }} / \mathrm{cm}^{-1}: 3340(\mathrm{NH}), 3025$ (arom. CH), 2910 (aliph. $\mathrm{CH}$ ), $2185(\mathrm{C} \equiv \mathrm{N}), 1732(\mathrm{C}=\mathrm{O}$; thiazoldinone) and $1646(\mathrm{C}=\mathrm{O}$; amide); ${ }^{1} \mathrm{H}$ NMR (DMSO- $\left.d_{6}\right) \delta / p p m: 1.03\left(\mathrm{t}, 3 \mathrm{H}, \mathrm{CH}_{3}, J=6 \mathrm{~Hz}\right.$ ), $2.04\left(\mathrm{p}, 2 \mathrm{H}, \mathrm{CH}_{2}, J=6 \mathrm{~Hz}\right), 4.17\left(\mathrm{~d}, 2 \mathrm{H}, \mathrm{C}_{2}-\mathrm{NH}, J=6 \mathrm{~Hz}\right), 4.39$ (t, $1 \mathrm{H}, \mathrm{CH}, J=6 \mathrm{~Hz}), 6.08(\mathrm{~d}, 1 \mathrm{H}$, furan- $\mathrm{H} 3, J=3.3 \mathrm{~Hz}), 6.33(\mathrm{t}$, $1 \mathrm{H}$, furan- $\mathrm{H} 4, \mathrm{~J}=3 \mathrm{~Hz}), 7.35-7.69(\mathrm{~m}, 12 \mathrm{H}, \mathrm{Ar}-\mathrm{H}+$ furan- $\mathrm{H} 5+$ $\mathrm{NH})$. MS (El, $70 \mathrm{ev}): \mathrm{m} / \mathrm{z}(\%)=542\left(\mathrm{M}^{+}, 25.5\right), 462(23), 278$ (12.5), 249 (100), 234 (8), 179 (28), 147 (32), 103 (69), 77 (100), 64 (34). Anal. Calcd. for $\mathrm{C}_{28} \mathrm{H}_{22} \mathrm{~N}_{4} \mathrm{O}_{4} \mathrm{~S}_{2}$ (542.63): C, $61.98 ; H, 4.09 ; \mathrm{N}, 10.33$. Found: $\mathrm{C}, 61.77 ; \mathrm{H}, 3.95 ; \mathrm{N}, 10.21 \%$.

\section{2,2'-(1,4-Phenylenebis(methanylylidene))bis(4-oxo-3- phenylthiazolidine-5,2-diylidene))bis(2-cyano- $N$-(furan-2- ylmethyl)acetamide) (16)}

To a solution of $7(0.02 \mathrm{~mol})$ in ethanol $(30 \mathrm{~mL})$, terephthalaldehyde $(0.01 \mathrm{~mol})$ and piperidine $(0.5 \mathrm{~mL})$ were added and the mixture was heated under reflux for 1 
$\mathrm{h}$; the solid product which was produced on heating was collected and recrystallized from dioxane as red solid. Yield (45\%), m.p. 290-292 ㅇ; IR (KBr) $v_{\max } / \mathrm{cm}^{-1}: 3341$ (br, NH), 3028 (arom. CH), 2926 (aliph. $\mathrm{CH}$ ), $2196(\mathrm{C} \equiv \mathrm{N}), 1712$ (C=O; thiazoldinone) and 1652 (C=O; amide); ${ }^{1} \mathrm{H}$ NMR (DMSO- $d_{6}$ )

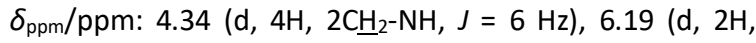
furan- $\mathrm{H} 3, J=6 \mathrm{~Hz}), 6.36(\mathrm{t}, 2 \mathrm{H}$, furan- $\mathrm{H} 4, J=6 \mathrm{~Hz}), 7.54-$ $8.11(\mathrm{~m}, 16 \mathrm{H}, \mathrm{Ar}-\mathrm{H}+$ furan-H5), $8.35(\mathrm{~s}, 2 \mathrm{H},=\mathrm{CH}), 10.06(\mathrm{t}$, $2 \mathrm{H}, 2 \mathrm{O}=\mathrm{C}-\mathrm{NH}-\mathrm{CH}_{2}, J=6 \mathrm{~Hz}$ ). MS (El, $\left.70 \mathrm{ev}\right) \mathrm{m} / \mathrm{z}(\%): 776\left(\mathrm{M}^{+}\right.$, 28.6), 662 (8), 501 (12.5), 439 (7.3), 385 (12), 242 (8), 149 (13), 85 (45), 71 (73), 57 (100). Anal. Calcd. for $\mathrm{C}_{42} \mathrm{H}_{28} \mathrm{~N}_{6} \mathrm{O}_{6} \mathrm{~S}_{2}$ (776.84): C, 64.94; H, 3.63; N, 10.82. Found: C, 64.72; H, $3.80 ; \mathrm{N}, 10.68 \%$.

\section{2-Cyano-N-(furan-2-ylmethyl)-2-(7-(4-methoxyphenyl)- 3,5-diphenyl-3,7-dihydro-2H-pyrano[2,3-d]thiazol-2- ylidene)acetamide(19)}

A mixture of $7(0.01 \mathrm{~mol})$, chalcone $21(0.01 \mathrm{~mol})$ and piperidine $(0.5 \mathrm{~mL})$ in ethanol $(30 \mathrm{~mL})$ was heated under reflux for $3 \mathrm{~h}$; the solid product which was produced on heating was collected and recrystallized from dioxane as yellow solid, Yield (40\%), m.p. 228-230 ㅇ; IR (KBr) $v_{\max } / \mathrm{cm}^{-1}: 3431$ (br, NH), 3022 (arom. CH), 2956 (aliph. CH), $2205(\mathrm{C} \equiv \mathrm{N})$, and 1651 (C=O; amide); ${ }^{1 \mathrm{H}}$ NMR (DMSO- $\left.d_{6}\right)$ $\delta /$ ppm: 3.43, $4.93(2 \mathrm{~d}, 2 \mathrm{H}$, pyran- $\mathrm{H} 4, \mathrm{H} 5, J=6 \mathrm{~Hz}), 3.84$ (s, $\left.3 \mathrm{H}, \mathrm{OCH}_{3}\right), 4.32\left(\mathrm{~d}, 2 \mathrm{H}, \mathrm{CH}_{2}-\mathrm{NH}, J=6 \mathrm{~Hz}\right), 6.09(\mathrm{~d}, 1 \mathrm{H}$, furan$\mathrm{H} 3, J=6 \mathrm{~Hz}), 6.30(\mathrm{t}, 1 \mathrm{H}$, furan- $\mathrm{H} 4, J=3 \mathrm{~Hz}), 6.85-7.47(\mathrm{~m}$, $16 \mathrm{H}, \mathrm{Ar}-\mathrm{H}+$ furan-H5), $8.20\left(\mathrm{t}, 1 \mathrm{H}, \mathrm{O}=\mathrm{C}-\mathrm{N} \underline{\mathrm{H}}-\mathrm{CH}_{2}, J=6 \mathrm{~Hz}\right.$ ). MS (El, 70 ev) m/z (\%): 559 (M+, 37.6), 502 (6), 435 (60.5), 392 (12), 313 (5), 217 (6), 105 (13), 84 (100), 81 (84), 57 (87). Anal. Calcd. for $\mathrm{C}_{33} \mathrm{H}_{25} \mathrm{~N}_{3} \mathrm{O}_{4} \mathrm{~S}$ (559.63): C, 70.82; $\mathrm{H}$, 4.50; N, 7.51. Found: C, 70.97; H, 4.36; N, $7.45 \%$.

\section{General Procedure for the Preparation of Compounds 23a and 23b:}

Method A: A mixture of $\mathbf{7}$ (0.01 mol), $\alpha$-cinnamonitrile $\mathbf{2 0}$ $(0.01 \mathrm{~mol})$ and piperidine $(0.5 \mathrm{~mL})$ in ethanol $(30 \mathrm{~mL})$ was heated under reflux for $1 \mathrm{~h}$; the solid product which produced on heating was collected and recrystallized from the proper solvent to give $\mathbf{2 3}$.

Method B: A mixture of compound $\mathbf{7}(0.02 \mathrm{~mol})$, aromatic aldehyde $(0.01 \mathrm{~mol})$ and piperidine $(0.01 \mathrm{~mol})$ in ethanol (30 $\mathrm{mL}$ ) was heated under reflux for $1 \mathrm{~h}$; the solid product which was produced on heating was collected and recrystallized from the proper solvent to give 23 .

2-(5-(2-Chlorobenzylidene)-4-oxo-3-phenylthiazolidin-2ylidene)-2-cyano- $\mathrm{N}$-(furan-2-ylmethyl)acetamide (23a) Orange powder (AcOH), Yield (50\%), m.p. 268-270 ㄷ; IR (KBr) $v_{\text {max }} / \mathrm{cm}^{-1}$ : 3325 (NH), 3060 (arom. CH), 2921 (aliph. $\mathrm{CH}), 2206(\mathrm{C} \equiv \mathrm{N}), 1717$ (C=O; thiazoldinone) and 1647 ( $\mathrm{C}=\mathrm{O}$; amide); ${ }^{1} \mathrm{H}$ NMR (DMSO- $d_{6}$ ): $\delta / p p m: 4.31$ (d, $2 \mathrm{H}, \mathrm{CH}_{2}-\mathrm{NH}, J$ $=6 \mathrm{~Hz}), 6.19(\mathrm{~d}, 1 \mathrm{H}$, furan- $\mathrm{H} 3, \mathrm{~J}=3 \mathrm{~Hz}), 6.35(\mathrm{t}, 1 \mathrm{H}$, furan-
$\mathrm{H} 4, \mathrm{~J}=3 \mathrm{~Hz}), 7.55-7.80(\mathrm{~m}, 10 \mathrm{H},+\mathrm{Ar}-\mathrm{H}+$ furan-H5), 7.89 $(\mathrm{s}, 1 \mathrm{H},=\mathrm{CH}), 8.28\left(\mathrm{t}, 1 \mathrm{H}, \mathrm{O}=\mathrm{C}-\mathrm{N} \underline{\mathrm{H}}-\mathrm{CH}_{2}, J=6 \mathrm{~Hz}\right) . \mathrm{MS}(\mathrm{El}, 70$ ev) $m / z(\%): 461\left(\mathrm{M}^{+}, 8.10\right), 426$ (50.6), 364 (15.5), 303 (22), 265 (19.5), 168 (88), 96 (90), 77 (100), 64 (43). Anal. Calcd. for $\mathrm{C}_{24} \mathrm{H}_{16} \mathrm{ClN}_{3} \mathrm{O}_{3} \mathrm{~S}$ (461.92): C, 62.41; $\mathrm{H}, 3.49 ; \mathrm{N}, 9.10$. Found: $\mathrm{C}, 62.35 ; \mathrm{H}, 3.54 ; \mathrm{N}, 9.00 \%$.

2-(5-(2-Bromobenzylidene)-4-oxo-3-phenylthiazolidin-2ylidene)-2-cyano- $\mathrm{N}$-(furan-2-ylmethyl)acetamide (23b) Orange powder (AcOH), Yield (52 \%), m.p. 265-266 ㄷ; IR $(\mathrm{KBr}) v_{\max } / \mathrm{cm}^{-1}$ : $3332(\mathrm{NH}), 3058$ (arom. $\left.\mathrm{CH}\right), 2921$ (aliph. $\mathrm{CH}), 2205(\mathrm{C} \equiv \mathrm{N}), 1714(\mathrm{C}=\mathrm{O}$; thiazoldinone) and $1647(\mathrm{C}=\mathrm{O}$; amide); ${ }^{1} \mathrm{H}$ NMR (DMSO- $\left.d_{6}\right): \delta / p p m: ~ 4.31\left(\mathrm{~d}, 2 \mathrm{H}, \mathrm{C}_{2}-\mathrm{NH}, J\right.$ $=6 \mathrm{~Hz}), 6.21(\mathrm{~d}, 1 \mathrm{H}$, furan- $\mathrm{H} 3, J=6 \mathrm{~Hz}), 6.36(\mathrm{t}, 1 \mathrm{H}$, furan$\mathrm{H} 4, J=3 \mathrm{~Hz}), 7.24-7.54(\mathrm{~m}, 10 \mathrm{H},+\mathrm{Ar}-\mathrm{H}+$ furan-H5), 8.11 $(\mathrm{s}, 1 \mathrm{H},=\mathrm{CH}), 12.26\left(\mathrm{t}, 1 \mathrm{H}, \mathrm{O}=\mathrm{C}-\mathrm{NH}-\mathrm{CH}_{2}, J=6 \mathrm{~Hz}\right) . \mathrm{MS}(\mathrm{El}, 70$ ev) $\mathrm{m} / \mathrm{z}(\%): 506\left(\mathrm{M}^{+}, 62.5\right), 507\left(\mathrm{M}^{+1}, 61.5\right), 426(30.6), 364$ (29.5), 303 (22), 265 (19.5), 212 (30), 133 (44), 96 (93), 77 (100), 53 (61). Anal. Calcd. for $\mathrm{C}_{24} \mathrm{H}_{16} \mathrm{~N}_{3} \mathrm{BrO}_{3} \mathrm{~S}$ (506.37): C, $56.93 ; \mathrm{H}, 3.18 ; \mathrm{N}, 8.30$. Found: $\mathrm{C}, 56.70 ; \mathrm{H}, 3.00 ; \mathrm{N}, 8.10 \%$.

\section{Antimicrobial Screening}

The disks of Whatman filter paper were prepared with standard size ( $6.0 \mathrm{~mm}$ diameter) and kept into $1.0 \mathrm{Oz}$ screw capped wide mouthed containers for sterilization. These bottles are kept into hot air oven at a temperature of 150 oC. Then, the standard sterilized filter paper disks impregnated with a solution of the test compound in DMF (100 $\mu \mathrm{L}, 5 \mathrm{mg} / \mathrm{mL}$ ) were placed on nutrient agar plate seeded with the appropriate test organism in triplicates. Standard concentrations of $10^{6} \mathrm{CFU} / \mathrm{mL}$ (Colony Forming Units $/ \mathrm{mL}$ ) and $10^{4} \mathrm{CFU} / \mathrm{mL}$ were used for antibacterial and antifungal assay, respectively. Pyrex glass Petri dishes $(9 \mathrm{~cm}$ in diameter) were used and two disks of filter paper were inoculated in each plate. The utilized test organisms were $S$. aureus, $S$. epidermidis and $B$. subitilis as examples of Gram-positive bacteria and $P$. aeruginosa, $P$. vulgaris and $K$. pneumonia as examples of Gram-negative bacteria. They were also evaluated for their in vitro antifungal potential against $A$. fumigatus, $A$. clavatus and $G$. candidium fungal strain. Ampicillin and gentamycin were used as standard antibacterial agents; while amphotericin B was used as standard antifungal agent. DMF alone was used as control at the same above-mentioned concentration and due this there was no visible change in bacterial growth. The plates were incubated at 37 ㅇ for $24 \mathrm{~h}$ for bacteria and for $48 \mathrm{~h}$ at 25 ㅇ for fungi. The mean zone of inhibition measured in $\mathrm{mm} \pm$ standard deviation beyond well diameter $(6 \mathrm{~mm})$ produced on a range of environmental and clinically pathogenic microorganisms. Compounds that showed significant growth inhibition zones using the twofold serial dilution technique were further evaluated for their minimal inhibitory concentrations (MICs). 


\section{Minimal Inhibitory Concentration (MIC) Measurement}

The microdilution susceptibility test in Müller-Hinton Broth (Oxoid) and Subouraud Liquid Medium (Oxoid) was used for the determination of antibacterial and antifungal activity, respectively. Stock solutions of the tested compounds, ampicillin, gentamycin, amphotericin B and sulfisoxazole were prepared in DMF at concentrations $1000 \mu \mathrm{g} / \mathrm{mL}$. Each stock solution was diluted with standard method broth (Difco) to prepare serial twofold dilutions in the range of $500-0.007 \mu \mathrm{g} / \mathrm{mL}$. $10 \mathrm{~mL}$ of the broth containing about $10^{6} \mathrm{CFU} / \mathrm{mL}$ of test bacteria or $10^{4}$ $\mathrm{CFU} / \mathrm{mL}$ of the test fungus was added to each well of 96well microtiter plate. The sealed microplates were incubated at 37 ㅇ for $24 \mathrm{~h}$ for antibacterial activity and at 25 ㅇ $\mathrm{C}$ for $48 \mathrm{~h}$ for antifungal activity in a humid chamber. At the end of the incubation period, the minimal inhibitory concentrations (MIC) values were recorded as the lowest concentrations of the substance that had no visible turbidity. Control experiments with DMF and uninoculated media were run parallel to the test compounds under the same conditions.

\section{REFERENCES}

[1] M. W. Khan, M. J. Alam, M. A. Rashid, R. Chowdhury, Bioorg. Med. Chem. 2005, 13, 4796.

[2] S. Onca, M. Punar, H. Erakosy, Chemotherapy 2004 50, 98.

[3] S. J. Kashyap, V. K. Garg, P. K. Sharma, N. Kumar, R. Dudhe, J. K. Gupta, Med. Chem. Res. 2012, 21, 2123.

[4] K. D. Hargrave, F. K. Hess, J. T. Oliver, J. Med. Chem. 1983, 26, 1158.

[5] W. C. Patt, H. W. Hamilton, M. D. Taylor, M. J. Ryan, D. G. Taylor Jr., C. J. C. Connolly, A. M. Doherty, S. R. Klutchko, I. Sircar, B. A. Steinbaugh, B. L. Batley, C. A. Painchaud, S. T. Rapundalo, B. M. Michniewicz, S. C. J. Olso, J. Med. Chem. 1992, 35, 2562.

[6] R. N. Sharma, F. P. Xavier, K. K. Vasu, S. C. Chaturvedi, S. S. Pancholi, J. Enzym. Inhib. Med. Chem. 2009, 24, 890.

[7] J. C. Jaen, L. D. Wise, B. W. Caprathe, H. Tecle, S. Bergmeier, C. C. Humblet, T. G. Heffner, L. T. Meltzner, T.A. Pugsley, J. Med. Chem. 1990, 33, 311.

[8] K. Omar, A. Geronikoki, P. Zoumpoulakis, C. Camoutsis, M. Sokovic, A. Ciric, J. Glamoclija, Bioorg. Med. Chem. 2010, 18, 426.

[9] K. Liaras, A. Geronikoki, J. Glamoclija, A. Ciric, M. Sokovic, Bioorg. Med. Chem. 2011, 19, 3135.

[10] F. W. Bell, A. S. Cantrell, M. Hogberg, S. R. Jaskunas, N. G. Johansson, C. L. Jordon, M. D. Kinnick, P. Lind, J. M. Morin Jr, R. Noreen, B. Oberg, J. A. Palkowitz, C. A. Parrish, P. Pranc, C. Sahlberg, R. J. Ternansky, R. T. Vasileff, L. Vrang, S. J. West, H. Zhang, X. X. Zhou, J. Med. Chem. 1995, 38, 4929.
[11] N. Ergenc, N.S. Capan, G. Gunay, S. Ozkirimli, M. Gungor, S. Ozbey, E. Kendi, Arch. Pharm. Med. Chem. 1999, 332, 343.

[12] J. S. Carter, S. Kramer, J. J. Talley, T. Penning, P. Collins, M. J. Graneto, K. Seibert, C. Koboldt, J. Masferrer, i. B. Zwe, Bioorg. Med. Chem. Lett. 1999, 9, 1171.

[13] A. Badorc, M. F. Bordes, P. De Cointet, P. Savi, A. Bernat, A. Lale, M. Petitou, J. P. Maffrand, J. M. Herbert, J. Med. Chem. 1997, 40, 3393.

[14] J. Rudolph, H. Theis, R. Hanke, R. Endermann, L. Johannsen, F. U. Geschke, J. Med. Chem. 2001, 44, 619.

[15] T. Wakita, K. Kinoshita, E. Yamada, Pest Manag. Sci. 2003, 59, 1016.

[16] Y. Shimokawatoko, K. Yamada, JP Patent, 2006, 131533.

[17] S. S. Pelosi, US Patent, 1976, 3946049.

[18] H. A. Burch, R. E. White, G. C. Wright, J. Pharm. Sci. 1980, 69, 107.

[19] H. S. A. Elzahabi, M. A. Salem, H. KH. Thabet, Der Phar. Chemica. 2011, 3, 48.

[20] M. H. Helal, S. Y. Abbas, M. A. Salem, A. A. Farag, Y. A. Ammar, Med. Chem. Res. 2013, 22, 5598.

[21] M. H. Helal, M. A. Salem, M. S. A. El-Gaby, M. Aljahdalif, Eur. J. Med. Chem. 2013, 65, 517.

[22] Y. A. Ammar, M. S. A. El-Gaby, M. A. Salem, Arab. J. Chem. 2014, 7, 615.

[23] M. H. Helal, S. A. El-Awdan, M. A. Salem, T. A. Abdelaziz, Y. A. Moahamed, A. A. El-Sherif, G. A. M. Mohamed, Spectrochim. Acta 2015, 135, 764.

[24] M. H. Helal, M. A. Salem, M. A. Gouda, N. S. Ahmed, A. A. El-Sherif, Spectrochim. Acta 2015, 147, 73.

[25] M. A. Salem, M. H. Helal, T. M. A. Eldebss, T. A. Abdelaziz, A. A. El-Sherif, G. A. M. Mohamed, J. Iran. Chem. Soc. 2015, 12, 1693.

[26] M. A. Salem, Der Phar. Chemica 2016, 8, 363.

[27] K. Konstantinos, Patent Schrift 1984, 646, 418; Chem. Abstr. 1985, 102, 184812v.

[28] L. Cantin, S. Choi, R. B. Clark, M. F. Hentemann, X. Ma, J. Rudolph, PCT Int. Appl. WO. 2004, 58, 174; Chem. Abstr. 2004, 141, 123483x.

[29] S. M. Z. Eldin, Naturforschung 1999, 54, 674.

[30] F. M. A. Razek, F. A. Michael, A. E. Mohamed, Arch. de. Pharm. 2006, 339, 305.

[31] R. E. Cooper, Analytical Microbiology, Ed. F.W. Kavangeh, 1\&2, Academic press, New York and London, 1972.

[32] A. H. Shamroukh, M. E. A. Zaki, E. M. H. Morsy, F. M. Abdel-Motti, F. M. E. Abdel-Megeid, Arch. Pharm. Chem. Life Sci. 2007, 340, 345.

[33] B. G. Tweedy, Phytopathol. 1964, 55, 910.

[34] A. A. El-Sherif, Inorg. Chim. Acta 2009, 362, 4991.

[35] A. A. El-Sherif, M. R. Shehata, M. M. Shoukry, M. H. Barakat, Spectrochim. Acta 2012, 96, 889. 